\title{
Performance of One-Hop/Symbol FHMA for Cellular Mobile Communications
}

\author{
Jiangzhou Wang, Senior Member, IEEE
}

\begin{abstract}
This paper is concerned with the bit-error rate (BER) performance of a cellular one-hop/symbol frequency-hopping multiple-access (FHMA) system operating through a multipath Rayleigh fading channel. $M$-ary frequency shift keying modulation with noncoherent square-law envelope demodulation and Reed-Solomon (RS) coding is considered. The multiple-access and adjacent cell interference of the cellular FHMA system has been studied. In order to illustrate how sensitive both systems are to the near/far problem, performance of the FHMA system is compared with that of a direct-sequence code-division multiple-access system for an equal system bandwidth. Also, this paper investigates the effect of the values of frequency reuse factor $(N)$ on the system capacity of the cellular frequency-hopping system.
\end{abstract}

Index Terms-Code-division multiple-access (CDMA), frequency hopping (FH), multipath fading channels.

\section{INTRODUCTION}

$\mathbf{R}$ ECENTLY, there has been an increased interest in the design and performance analysis of spread-spectrum systems. Among several other qualities, these systems can combat multipath fading and provide multiple-access capability. All direct sequence (DS), frequency hopping (FH), and hybrid DS/FH schemes have been proposed for this use [1]-[13]. The main purpose of using DS modulation is that instead of regarding the multipath phenomenon as a disturbance that needs to be suppressed, it should be regarded as an opportunity to improve system performance [1]. This can be done by considering the explicit diversity structure of resolvable paths (i.e., spread-spectrum diversity) and by optimally combining the contribution from different paths. A basic disadvantage of DS modulation is the need for power control due to the near/far problem. The use of frequency-hopping multiple-access schemes [2]-[12] has been proposed as an alternative to frequency-division multiple-access (FDMA) techniques to guard against interference from other users.

The performance of frequency-hopping multiple-access (FHMA) over Rayleigh fading channels with a single cell was extensively analyzed in the past years. Reference [12] proposed a multihop-per-symbol FHMA system for indoor wireless communications. Continuing the previous research on performance analysis of single-cell FHMA systems, we consider multiple-cell FH systems. It has been shown in [12] that in a multihop-per-symbol FHMA system, performance improves when the number of hops per symbol increases due to multihop

Manuscript received March 19, 1999; revised October 2, 2000. This work was supported by the Research Grant Council (RGC) of the Hong Kong Government.

The author is with the Department of Electrical and Electronic Engineering, University of Hong Kong, Hong Kong (e-mail: jwang@eee.hku.hk).

Publisher Item Identifier S 0018-9545(01)01225-7. diversity. However, the multihop system is very complicated in implementation due to the use of high-speed frequency synthesizers. On the other hand, in a slow FH system, a very large buffer is required to realize interhop interleaving techniques (whereby each bit of a codeword is transmitted during a hop to reduce the effect of hits from nonreference users), which is used in conjunction with error-correcting coding. Therefore, one hop per symbol is proposed in our study. The bit-error-rate (BER) performance of one hop per symbol FHMA systems employing noncoherent square-law envelope detection and Reed-Solomon (RS) coding is studied for a cellular mobile channel. The hopping instants of the various users are not aligned in time. For FH systems, it is natural to consider $M$-ary frequency-shift keying (MFSK) $(M=2,4,8, \ldots)$ as a candidate modulation scheme. Reed-Solomon codes are preferable in nonbinary systems because of their good burst-error correcting capability [3].

\section{SyStem Models}

\section{A. Transmitter Model}

The transmitter model of a FH system consists of a serial-toparallel converter, a RS encoder, an interleaver, an MFSK modulator, and a frequency hopper. The transmitted signal of the $k$ th user in the FHMA system takes the following form:

$$
\begin{aligned}
S_{k}(t)=\operatorname{Re}\left\{\sqrt { 2 \widehat { P } _ { k } } \operatorname { e x p } \left\{j 2 \pi \left[f_{0}+f_{c_{k}}+f_{k}(t)\right.\right.\right. \\
\\
\left.\left.\left.+b_{k}(t) \Delta\right] t+j \alpha_{k}(t)\right\}\right\}
\end{aligned}
$$

where

\section{$\operatorname{Re}\{\bullet\} \quad$ real part;}

$\widehat{P}_{k} \quad$ transmitted power of the $k$ th user;

$f_{0} \quad$ carrier frequency, which is common to all users;

$f_{c_{k}} \quad$ frequency of the $c_{k}$ th cell, which belongs to the set $\left\{F_{1}, \ldots, F_{N}\right\}$, whose frequency reuse factor is $N$; $f_{k}(t) \quad$ hopping pattern of the $k$ th user and takes on a constant value $f_{k}^{(\lambda)}$ during the $\lambda$ th hop, which belongs to the set $\left\{\nu_{1}, \ldots, \nu_{q}\right\}$ of $q$ not necessarily equally spaced frequencies with minimum spacing $W$.

It is assumed that $\left\{f_{k}^{(\lambda)}\right\}$ is a first-order Markov sequence, so that two consecutive hopping frequencies are always different. It is assumed that the duration of a single hopping interval (dwell time) equals one symbol duration (i.e., one hop per symbol). The phase $\alpha_{k}(t)$ is introduced by the MFSK modulator and takes on a constant value $\alpha_{k}^{(\lambda)}$ during $\lambda$ th coded symbol; $b_{k}(t)$ is the interleaved sequence of RS coded symbols; the $\lambda$ th symbol of $b_{k}(t)$ has amplitude $b_{k}^{(\lambda)}$, taking values from 
$\{ \pm 1, \pm 3, \ldots, \pm(M-1)\}$, where $M$ is restricted to be a power of two (one symbol corresponds to $\log _{2} M$ bits). The coded symbol rate and coded symbol duration are given by $R_{s}=R_{b} / \log _{2} M$ and $T_{s}=1 / R_{s}=T_{b} \log _{2} M$, respectively, where $R_{b}$ and $T_{b}$ denote the rate and duration of the coded bits $\left(T_{b}=1 / R_{b}\right)$. The information bit rate (i.e., the rate of source bits) is given by $R_{i}=R_{b} m / n$, where $m / n$ is the rate of the RS code. During a given hop interval, one of $M$ possible signals is transmitted. The $M$ signals are sinusoidal tones of duration $T_{s}$ with frequency spacing of $2 \Delta$. In order that these tones be orthogonal when aligned in time, $2 \Delta T_{s}$ must be integer $2 \Delta T_{s}=n_{o}$. However, multiple-access interference signals are not time aligned with the useful signal, partially due to multipath propagation. Assuming a misalignment that is uniformly distributed over a symbol interval, the root mean square correlation between adjacent tones equals $\left(\sqrt{2} \pi n_{0}\right)^{-1}$. Subsequently, it will be assumed that $n_{O}$ is sufficiently large to ignore the correlation between tones at different frequencies. The resulting cellular system bandwidth is given by

$$
\begin{aligned}
B & =2 \Delta M q N=M q N\left(n_{0} / T_{s}\right)=M q N n_{0} /\left(T_{b} \log _{2} M\right) \\
& =M q N n_{0}(n / m) /\left(T_{i} \log _{2} M\right)
\end{aligned}
$$

where $T_{i}$ denotes the duration of the source (uncoded) bits.

\section{B. Channel Model}

It is assumed that the cellular channel between the $k$ th user and the corresponding receiver at the base station of the cell of interest is a multipath Rayleigh fading channel [13]. The multipath Rayleigh fading channel between the $k$ th user and receiver of interest (namely, the receiver in the base station of what we refer to as the first cell) is modeled by the complex lowpass equivalent impulse response

$$
h_{k}(t)=\frac{1}{\left(d_{1, k}\right)^{\gamma / 2}} \sum_{l=1}^{L} \beta_{k l} \exp \left(j \mu_{k l}\right) \delta\left(t-\tau_{k l}\right)
$$

where $d_{1, k}\left(d_{1, k} \neq 0\right)$ is the distance between the $k$ th user and the base station of the first cell and $\gamma$ is the propagation path loss exponent. The random gain $\beta_{k l}$ and random phase $\mu_{k l}$ of the fading component of the $l$ th path of the $k$ th user have a Rayleigh distribution with $E\left[\beta_{k l}^{2}\right]=2 \rho_{k l}=2 \rho$ for all $k$ and $l$ and a uniform distribution in $[0,2 \pi]$, respectively. The path delay $\tau_{k l}$ is uniformly distributed in $\left[0, T_{s}\right]$. We assume that there are $L$ paths associated with each user. The gains, delays, and phases of different paths and/or of different users are all statistically independent. Also, the channel introduces additive white Gaussian noise $n(t)$ with two-sided power spectral density $N_{o} / 2$. Hence, the received signal can be represented as

$$
\begin{gathered}
r(t)=\operatorname{Re}\left\{\sum_{k=1}^{C K} \sqrt{2 P_{k} \xi\left(\gamma, c_{k}, k\right)} \sum_{l=1}^{L} \beta_{k l} \exp \left[j \psi_{k l}(t)\right]\right. \\
\cdot \exp \left\{j 2 \pi \left[f_{0}+f_{c_{k}}+f_{k}\left(t-\tau_{k l}\right)\right.\right. \\
\left.\left.\left.+b_{k}\left(t-\tau_{k l l}\right) \Delta\right] t\right\}\right\}+n(t)
\end{gathered}
$$

where $\psi_{k l}(t)=\mu_{k l}+\alpha_{k}\left(t-\tau_{k l}\right)-2 \pi\left[f_{0}+f_{c_{k}}+f_{k}(t-\right.$ $\left.\left.\tau_{k l}\right)+b_{k}\left(t-\tau_{k l}\right) \Delta\right] \tau_{k l}$ and $C$ stands for the number of cells, each one containing $K$ active users, and $c_{k}$ denotes the $c_{k}$ th cell [the integer portion of $1+(k-1) / K, c_{k}=1, \ldots, C$ ]. The first cell $\left(c_{k}=1\right)$ is defined as the cell of interest, and $P_{k}$ and $\xi\left(\gamma, c_{k}, k\right)$ are defined as

$$
\begin{aligned}
P_{k} & =\widehat{P}_{k} /\left(d_{c_{k}, k}\right)^{\gamma} \\
\xi\left(\gamma, c_{k}, k\right) & =\left(d_{c_{k}, k} / d_{1, k}\right)^{\gamma}
\end{aligned}
$$

respectively, where $d_{c_{k}, k}$ is the distance of the $k$ th mobile user to its own base station (the $c_{k}$ th cell) and $d_{c_{k}, k} \neq 0$.

\section{Receiver Model}

The receiver consists of the following: a frequency dehopper, MFSK demodulator, hard decision device, deinterleaver, RS decoder, and a parallel-to-serial converter. A detailed model of the frequency dehopper and the MFSK demodulator is shown in Fig. 1. The received signal $r(t)$ enters a bandpass filter that removes out of band noise. The mixer of the dehopper performs the appropriate frequency translation, according to both the first-cell frequency $F_{1}$ and the hopping sequence $f_{i}(t)$ of user $i$. It is assumed that the hopping pattern of the receiver is synchronized with the hopping pattern of the signal associated with $j$ th path of user $i$ (denoted as the reference path). The bandpass filter that follows the mixer removes both high-frequency terms and terms corresponding to nonreference user hopping frequency. The dehopper output signal is given by

$$
\begin{aligned}
r_{d}(t)= & \sum_{k=1}^{C K} \sqrt{2 P_{k} \xi\left(\gamma, c_{k}, k\right)} \sum_{l=1}^{L} \\
& \cdot \beta_{k l} \delta\left[f_{c_{k}}+f_{k}\left(t-\tau_{k l}\right), F_{1}+f_{i}\left(t-\tau_{i j}\right)\right] \\
& \cdot \cos \left\{2 \pi\left[f_{0}+b_{k}\left(t-\tau_{k l}\right) \Delta\right] t+\Psi_{k l l}(t)\right\}+\hat{n}(t)
\end{aligned}
$$

where

$\delta(x, y) \quad$ Kronecker function, defined as $\delta(x, y)=1$ or 0 for $x=y$ or $x \neq y$, respectively;

$F_{1} \quad$ frequency of the first cell (or the cell of interest); $\hat{n}(t) \quad$ can be treated as bandlimited Gaussian noise with spectral density $N_{0} / 2$.

The phase waveform $\Psi_{k l}(t)=\psi_{k l}(t)-z_{i}(t)$; where $z_{i}(t)$ is the phase introduced by the dehopper that is constant over symbol interval, $z_{i}^{(\lambda)}$ stands for the constant phase during the $\lambda$ th symbol. Note that the dehopper suppresses, at any instant $t$, all path signals whose hopping frequency at instant $t$ differs from $F_{1}+f_{i}\left(t-\tau_{i j}\right)$. The reference path signal is not suppressed. The other path signals from the reference user are suppressed during a part of a symbol, depending on the relative delay of the considered path with respect to the reference path. Path signals from the users of the first cell contribute the dehopper output during those time intervals for which their hopping frequencies accidentally equal that of the reference path signal. When the frequency reuse factor $N=7$ [see Fig. 2(a)], there is no multiple-access (or adjacent-cell) interference from all cells of the first and second layers and 12 of 18 cells of the third layer since all their frequencies are different from $F_{1}$. However, the frequencies of the remaining six (shadowed cells) 


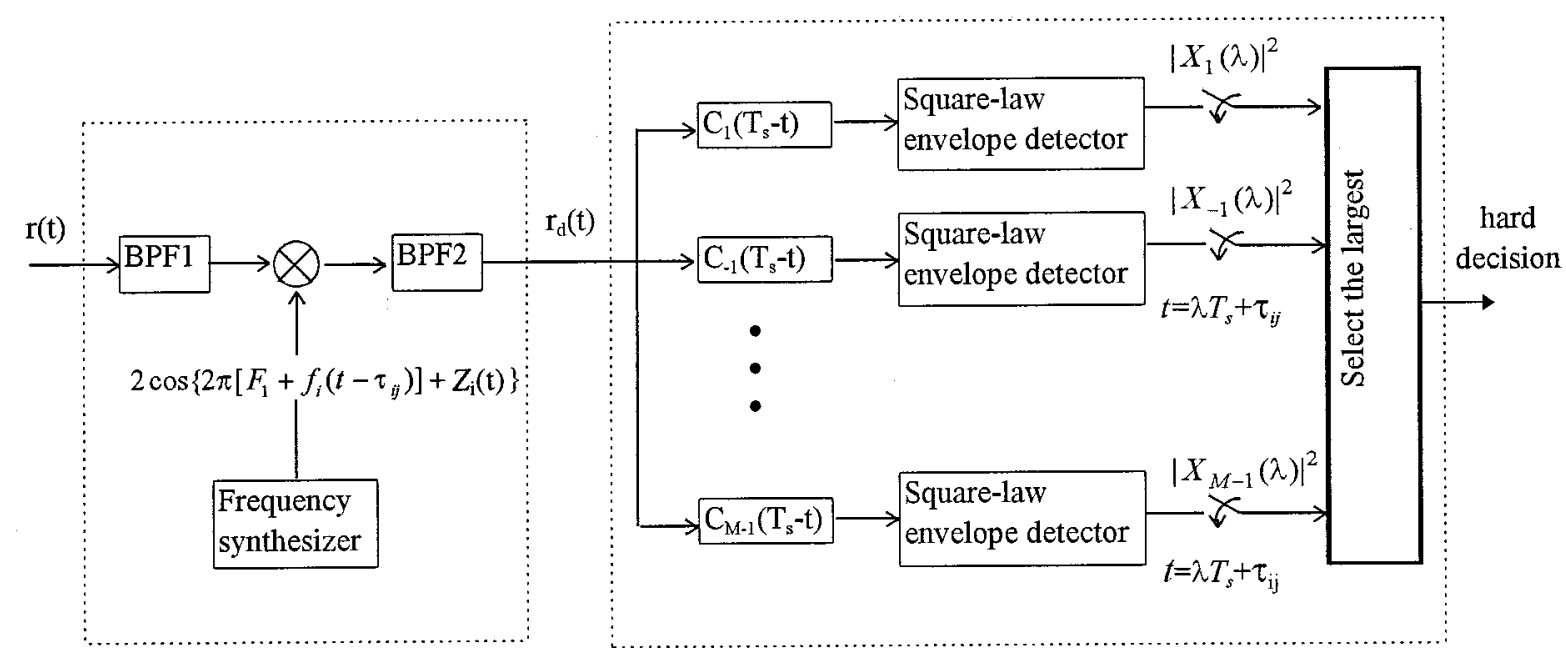

Frequency-dehopper

Fig. 1. Detailed model of frequency dehopper and MFSK demodulator.

out of 18 cells of the third layer are the same as $F_{1}$. Therefore, the six cells may cause multiple-access interference, when their hopping frequencies accidentally equal that of the reference path signal. On the other hand, when the frequency reuse factor $N=3$ [see Fig. 2(b)], there is no multiple-access (or adjacent-cell) interference from all cells of the first layer, six of 12 cells of the second layer, and 12 of 18 cells of the third layer since their frequencies are different from $F_{1}$. However, the remaining six of 12 cells of the second layer and six of 18 cells of the third layer may cause multiple-access interference, since their cell frequencies are the same as $F_{1}$.

The dehopper output signal $r_{d}(t)$ enters a noncoherent MFSK demodulator. It consists of $M$ bandpass matched filters with impulse responses $\left\{C_{\varepsilon}\left(T_{s}-t\right)\right\}$, where $C_{\varepsilon}(t)=2 \cos \left[2 \pi\left(f_{0}+\varepsilon \Delta\right) t\right], 0<t<T_{s}$ and $\varepsilon= \pm 1, \pm 3, \ldots, \pm(M-1)$, followed by square-law envelope detectors. A square-law envelope detector consists of a squarer followed by a lowpass filter; the output of the lowpass filter is the square of the envelope of the bandpass signal at the input of the squarer. The $M$ square-law detector outputs are sampled at the instants $\lambda T_{s}+\tau_{i j}$; this yields the $M$ random variables $\left\{\left|X_{\varepsilon}(\lambda)\right|^{2}\right.$ for $\varepsilon= \pm 1, \pm 3, \ldots, \pm(M-1)$, where

$$
X_{\varepsilon}(\lambda)=\int_{(\lambda-1) T_{s}+\tau_{i j}}^{\lambda T_{s}+\tau_{i j}} 2 r_{d}(t) \exp \left[j 2 \pi\left(f_{0}+\varepsilon \Delta\right) t\right] d t
$$

The receiver bases its hard decision about the coded symbol $b_{i}^{(\lambda)}$ on the $M$ random variables $\left\{\left|X_{\varepsilon}(\lambda)\right|^{2}\right\}$ for $\varepsilon= \pm 1, \pm 3, \ldots, \pm(M-1)$, by selecting the largest $\left|X_{\varepsilon}(\lambda)\right|^{2}$ and declaring that the symbol with the corresponding value of $\varepsilon$ has been transmitted.

\section{Statistical Description of $X_{\varepsilon}(\lambda)$}

In the following, it will be assumed that $E\left[\beta_{k l}^{2}\right]$ equals $2 \rho$ for all $k$ and $l$. The random variable $X_{\varepsilon}(\lambda)$ given by (8) consists of the following terms:
MFSK demodulator

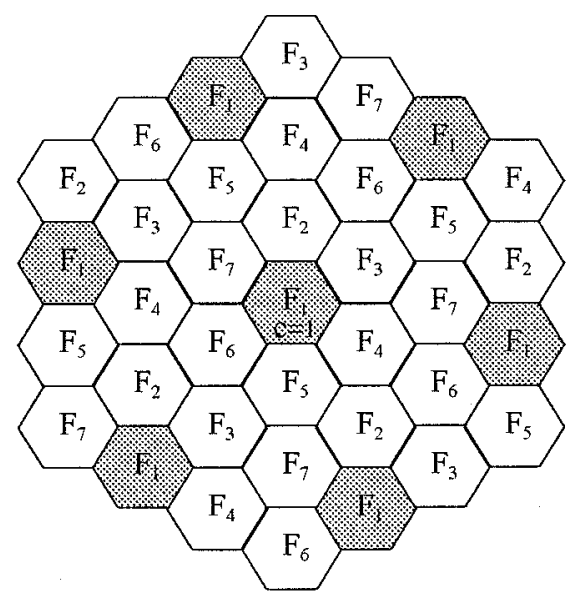

(a)

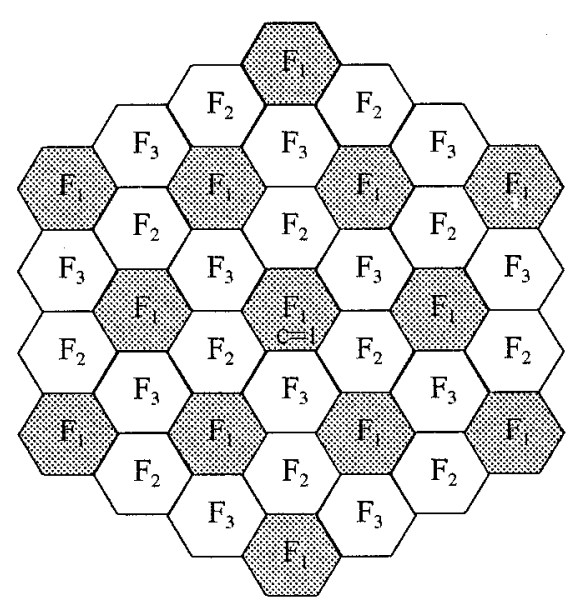

(b)

Fig. 2. The cellular model ( $N$ is frequency reuse factor).

1) A complex-valued reference path term $D_{j, \varepsilon}(\lambda)$, which is due to the $j$ th path signal from the reference user. As this reference path signal passes through the dehopper and only through the MFSK demodulator branch with $\varepsilon=b_{i}^{(\lambda)}$. 
This useful term is given by

$$
\begin{aligned}
& D_{j, \varepsilon}(\lambda) \\
& \quad=\sqrt{2 P_{i}}\left\{\beta_{i j} \exp [j V(i, j, \lambda)]\right\} T_{s} \delta\left(\varepsilon, b_{i}^{(\lambda)}\right)
\end{aligned}
$$

where $V(k, l, \zeta)$ is a phase angle and is defined as

$$
\begin{aligned}
& V(k, l, \zeta) \\
& =\mu_{k l}+\alpha_{k}^{(\zeta)}-z_{i}^{(\lambda)} \\
& +2 \pi\left[f_{0}+f_{c_{k}}+f_{k}^{(\zeta)}+b_{k}^{(\zeta)} \Delta\right] \tau_{k l} \\
& \quad-2 \pi\left[f_{0}+F_{1}+f_{i}^{(\lambda)}+b_{i}^{(\lambda)} \Delta\right] \tau_{i j} .
\end{aligned}
$$

Strictly speaking, the probability density function of $V(k, l, \zeta)$ should be triangular, since $V(k, l, \zeta)$ is a sum of uniform random variables. However, because for $\exp [j V(k, l, \zeta)]$ any value of $V(k, l, \zeta)$ outside [0, $2 \pi]$ is equivalent to a value inside $[0,2 \pi], V(k, l, \zeta)$ is assumed uniform over $[0,2 \pi]$. It is clear from (9) that $D_{j, \varepsilon}(\lambda)=0$ for $\varepsilon \neq b_{i}^{(\lambda)}$, and for $\varepsilon=b_{i}^{(\lambda)}$ the real and imaginary parts of $D_{j, \varepsilon}(\lambda)$ are uncorrelated and have the same variance $2 P_{i} \rho T_{s}^{2}$. This variance is obtained by averaging over the gain and the phase of the reference path signal. Therefore, the variance of the real and imaginary parts of the useful term is given by

$$
S_{1}=2 P_{i} \rho T_{s}^{2} \delta\left(\varepsilon, b_{i}^{(\lambda)}\right) .
$$

2) A complex-valued multipath term, which is due to the $L-1$ other path signals of the reference user. The contributions from different paths are uncorrelated.

3) A complex-valued multiple-access interference term, which is due to the path signals from the $K-1$ nonreference users of the cell of interest.

4) A complex-valued adjacent-cell interference term, which is due to the signals from the adjacent cells, whose frequencies are the same as the frequency $\left(F_{1}\right)$ of the cell of interest.

5) A complex-valued Gaussian noise term, which is due to the noise $\hat{n}(t)$ at the dehopper output. The real and imaginary parts of the noise term are uncorrelated and have the same variance $N_{0} T_{s}$. The noise contribution of different matched filter outputs are uncorrelated.

\section{A. The Multipath Term}

During the $\lambda$ th hop (symbol) of the reference path signal, part of the signal corresponding to the same hop of another path of the reference user, passes through the dehopper that is synchronized to the reference path signal. During the considered hop of the reference path signal, the signals corresponding to the next hop of an earlier path signal of the reference user or the previous hop of a later path signal of the reference user are suppressed by the dehopper. This is because two consecutive hopping frequencies are always different for the first-order Markov hopping sequence. The dehopper output signal passes through the same MFSK demodulator branch as the reference path signal [i.e., the branch with $\left.\varepsilon=b_{i}^{(\lambda)}\right]$, because they carry the same symbols. The contribution from the $l$ th path is given by

$$
\begin{aligned}
D_{l, \varepsilon}(\lambda)= & \sqrt{2 P_{i}} \beta_{i l} \\
& \cdot \exp [j V(i, l, \lambda)]\left(T_{s}-\left|\tau_{i l}-\tau_{i j}\right|\right) \delta\left(\varepsilon, b_{i}^{(\lambda)}\right)
\end{aligned}
$$

where $V(i, l, \lambda)$ is given by (10). It is known from the channel model that the path delay $\tau_{k l}$ for any $k$ and $l$ is uniformly distributed in $\left[0, T_{s}\right]$. Therefore, the delay difference between any two paths from the reference user $\tau_{i l}-\tau_{i j}$ has a symmetric, triangular distribution in $\left[-T_{s}, T_{s}\right]$. When $\varepsilon=b_{i}^{(\lambda)}$, the real and imaginary parts of $D_{l, \varepsilon}(\lambda)$ are uncorrelated and have the same variance, equal to $P_{i} \rho T_{s}^{2}$. This variance is obtained by averaging over the gain, the phase, and the delay difference (with respect to the reference path signal) of the nonreference path signal from the reference user. When $\varepsilon \neq b_{i}^{(\lambda)}, D_{l, \varepsilon}(\lambda)$ is identically zero. The variance of the real and imaginary part of the total multipath term in $X_{\varepsilon}(\lambda)$ is given by

$$
S_{2}=P_{i} \rho T_{s}^{2}(L-1) \delta\left(\varepsilon, b_{i}^{(\lambda)}\right) .
$$

The contribution of the multipath term to the nearest demodulator branch is shown in the Appendix with the variance equal to $\left(P_{i} \rho \cdot T_{s}^{2}\right) /\left(\pi^{2} \cdot n_{0}^{2}\right)$, where $n_{0}$ is an integer. When $n_{0} \geq 1$, this variance is much smaller than the variance of $D_{l, \varepsilon}(\lambda)$. Therefore, when the spacing between adjacent tones is sufficiently large $\left(n_{0} \geq 1\right)$, the contribution of the multipath term to other MFSK demodulator branches is negligible. Hence, the multipath term is a useful term.

\section{B. The Multiple-Access Interference from the Cell of Interest}

The contribution from the $l$ th path signal of nonreference user $k$ of the cell of interest for which the $\lambda$ th symbol starts earlier than the $\lambda$ th symbol of the reference path signal, is given by

$$
\begin{array}{r}
I_{k, l}(\varepsilon, \lambda) \\
=\sqrt{2 P_{k}}\left\{\beta _ { k l } \left\{\exp [j V(k, l, \lambda)] \delta\left[f_{k}^{(\lambda)}, f_{i}^{(\lambda)}\right] \delta\left[\varepsilon, b_{k}^{(\lambda)}\right]\right.\right. \\
\cdot\left[T_{s}-\left(\tau_{i j}-\tau_{k l}\right)\right]+\exp [j V(k, l, \lambda+1)] \\
\left.\left.\cdot \delta\left[f_{k}^{(\lambda+1)}, f_{i}^{(\lambda)}\right] \delta\left[\varepsilon, b_{k}^{(\lambda+1)}\right] \cdot\left(\tau_{i j}-\tau_{k l l}\right)\right\}\right\} .
\end{array}
$$

Fig. 3 schematically represents the $\lambda$ th hop of the reference path signal and the earliest and the latest path signals from nonreference user $k$. Let us consider the case where the $\lambda$ th hop of the reference user starts during the $\lambda$ th symbol of the earliest path signal from user $k$.

1) When the frequencies of the $(\lambda-1)$ th, $\lambda$ th, and $(\lambda+1)$ th hops of user $k$ are all different from the frequency of the $\lambda$ th hop of the considered reference path, there is no interference from user $k$.

2) When the frequency of the $\lambda$ th hop from user $k$ equals the frequency of the $\lambda$ th hop of the considered reference path signal, the considered reference path hop is partially hit by all $L$ path signals of user $k$. The interfering path signals pass through the branch of the MFSK demodulator with 
reference path hop

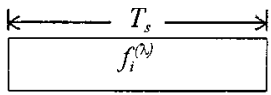

earliest path of user $k$

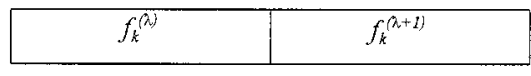

latest path of user $k$

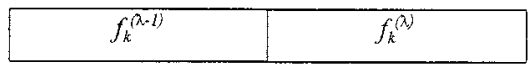

Fig. 3. Illustration of multiple-access interference.

$\varepsilon=b_{k}^{(\lambda)}$. When the spacing $2 \Delta$ between adjacent tones is sufficiently large, the effect on other MFSK demodulator branches can be ignored. The variance of the real (or imaginary) part of such interference from user $k$ is

$$
\sigma_{1}^{2}=P_{k} \rho T_{s}^{2} L \delta\left(\varepsilon, b_{k}^{(\lambda)}\right)
$$

Note that when $f_{k}^{(\lambda)}=f_{i}^{(\lambda)}$, neither $f_{k}^{(\lambda-1)}$ nor $f_{k}^{(\lambda+1)}$ is equal to $f_{i}^{(\lambda)}$, since two consecutive hopping frequencies are different. For a large number $(q)$ of available hopping frequencies, the probability of a hit from a nonreference user is small. Therefore, in the following, we shall consider only two dominating events and ignore the other events. The two dominating events are as follows.

1) The considered reference path symbol is not hit by any nonreference user from the cell of interest, the total multiple-access interference is similarly zero;

2) the considered reference path symbol is hit by only one nonreference user from the cell of interest; the joint occurrence of hits by more nonreference users has a much smaller probability than the occurrence of a single hit (roughly $1 / q^{2}$ and $2 / q$, respectively) and, therefore, will be ignored. There are $M$ single-hit events ( $M$ possible values for the nonreference symbol involved in the hit) and the probability of each such event is approximated by

$$
P_{h}=2(K-1) /(q M) .
$$

\section{The Adjacent-Cell Interference}

The contribution from the $l$ th path signal of user $k$ of an adjacent (or the $c_{k}$ th) cell, for which the $\lambda$ th symbol starts earlier than the $\lambda$ th symbol of the reference path signal of the first cell, is given by

$$
W_{k, l}(\varepsilon, \lambda)=\sqrt{\xi\left(\gamma, c_{k}, k\right)} I_{k, l}(\varepsilon, \lambda) \delta\left[f_{c_{k}}, F_{1}\right]
$$

where $\xi\left(\gamma, c_{k}, k\right)$ and $I_{k, l}(\varepsilon, \lambda)$ are given by (6) and (14), respectively. When the frequency of the $c_{k}$ th cell is equal to $F_{1}$ (the frequency of the first cell), there may be adjacent cell interference. Otherwise, there is no interference from the $c_{k}$ th cell. $W_{k, l}(\varepsilon, \lambda)$ has the conditional variance conditioned on $\xi\left(\gamma, c_{k}, k\right)$ given by

$\left.E\left[W_{k, l}^{2}(\varepsilon, \lambda)\right]\right|_{\xi\left(\gamma, c_{k}, k\right)}=\xi\left(\gamma, c_{k}, k\right) E\left[I_{k, l}^{2}(\varepsilon, \lambda)\right] \delta\left[f_{c_{k}}, F_{1}\right]$.

In order to average the variance over the area of the $c_{k}$ th cell, we approximate the hexagonal cell with a circular cell of equal area, as in [1]. As shown in Fig. 2(a), when the frequency reuse factor $N=7$, it is only possible for adjacent cell interference to be from six (shadowed cells) out of 18 third-layer cells, whose frequencies are the same as $F_{1}$. However, when the frequency reuse factor $N=3$, adjacent cell interference could be from six of 12 second-layer cells and six of 18 third-layer cells [see Fig. 2(b)]. Referring to [1, Table I], the average of $\xi\left(\gamma, c_{k}, k\right)$ over the areas of the shadowed cells, indicated in Fig. 2(a) and (b), is shown in Table I for different values of propagation exponent $(\gamma)$. Therefore, when the frequency of the $\lambda$ th hop from user $k$ equals the frequency of the $\lambda$ th hop of the considered reference path signal, the considered reference path hop is partially hit by all $L$ path signals of user $k$. The interfering path signals pass through the branch of the MFSK demodulator with $\varepsilon=b_{k}^{(\lambda)}$. The variance of the real (or imaginary) part of such interference from user $k$ is

$$
\sigma_{2}^{2}(\gamma, x)=P_{k} \rho T_{s}^{2} L \xi(\gamma, x) \delta\left(\varepsilon, b_{k}^{(\lambda)}\right)
$$

\section{BIT ERRor RATE}

It follows from Section III, that the real and imaginary parts of the complex-valued random variables $\left\{X_{\varepsilon}(\lambda) \mid \varepsilon= \pm 1, \ldots, \pm(M-1)\right\}$ are uncorrelated and have the same variance. This variance can be written as $S(\varepsilon)+\sigma^{2}(\varepsilon)$, where $S(\varepsilon)$ denotes the useful part, caused by the reference path signal term and the multipath term, and $\sigma^{2}(\varepsilon)$ is the contribution from the additive noise, the multiple access, and adjacent-cell interference. In Section IV-A, it will be assumed that the random variables $\left\{X_{\varepsilon}(\lambda) \mid \varepsilon= \pm 1, \ldots, \pm(M-1)\right\}$ are Gaussian, which will allow us to obtain analytical results in closed form. The Gaussian approximation is shown accurate [12] when the number of paths $L$ is large ( $L \gg 1$ ), because the channel parameters (gain, phase, delay) are assumed independent from path to path and because each multiple access interference (MAI) term (14) looks like Gaussian (Rayleigh gain and uniform phase).

\section{A. Symbol Error Probability Before Decoding}

The receiver bases its hard decision about the symbol $b_{i}^{(\lambda)}$ on the $M$ decision variables $\left|X_{\varepsilon}(\lambda)\right|^{2}(\varepsilon= \pm 1, \ldots, \pm(M-1))$. The receiver declares that the symbol corresponding to the largest $\left|X_{\varepsilon}(\lambda)\right|^{2}$ has been transmitted. The symbol error rate (SER) $P_{s}$ after hard decision is approximated by the union bound. Let us consider the case where $b_{i}^{(\lambda)}=1$. Then this approximation yields

$$
P_{s}=\sum_{\varepsilon \neq 1} \sum_{h \in H} P_{s}(\varepsilon \mid h) P_{h}(h)
$$

where

$$
P_{s}(\varepsilon \mid h)=\operatorname{Pr}\left[\left|X_{1}(\lambda)\right|^{2}<\left.X_{\varepsilon}(\lambda)\right|^{2} \mid h\right]
$$

$P_{h}(h)$ is the probability of even $h$ and $H$ is the set of the joint occurrence of the following considered events:

$$
H=\left\{h_{0}, h_{\varepsilon_{0}}, h_{\varepsilon_{1}}, \ldots, h_{\varepsilon_{I}} \mid h_{\varepsilon_{i}}= \pm 1, \ldots, \pm(M-1)\right\}
$$


TABLE I

ADJACENT CELl INTERFERENCE FOR A SHADOWEd CELL

\begin{tabular}{c|c|c|c}
\hline \multirow{2}{*}{$\gamma$} & \multicolumn{2}{|c|}{$N=3$} & \multirow{2}{*}{\begin{tabular}{c} 
\\
\cline { 2 - 4 }
\end{tabular}} \\
\cline { 2 - 4 } & $\xi(\gamma, 1)$ & $\xi(\gamma, 2)$ & third layer \\
\cline { 2 - 4 } & second layer & third layer & 0.0156 \\
\hline 2 & 0.049 & 0.0202 & 0.0023 \\
\hline 3 & 0.013 & 0.0033 & 0.0003 \\
\hline 4 & 0.0037 & 0.0006 & \\
\hline
\end{tabular}

In the above, $h_{0}$ is the no-hit event. Whereas $\left\{h_{\varepsilon_{i}}, i=\right.$ $0, \ldots, I\}$ are single-hit events both with $\varepsilon_{i}$ as the interfering symbol and $I$ as the total interfering cells; whereas $h_{\varepsilon_{0}}$ is a single-hit event from the cell of interest. When the frequency reuse factor of a cellular model $N=7, I=6$ [shadowed cells of Fig. 2(a)], whereas when $N=3$ [Fig. 2(b)], $I=12$.

For the joint occurrence of the events, detailed consideration at the outputs of the $M$ branches of the MFSK demodulator is very complicated, since there are too many combinations of the joint occurrence and the $M$ branches. In order to obtain simple analytical results we use the following approximation; all joint occurrences of two or more single-hit events are assumed with the identical interfering symbols (i.e., $\widehat{\varepsilon}=\varepsilon_{1}=\varepsilon_{2}=\cdots=$ $\left.\varepsilon_{I}\right)$. This assumption can be justified mathematically for interfering symbols from adjacent cells, based on the fact that interference from any adjacent cell with respect to useful signal power is much less than one (see Table I).

Suppose two events $h_{\varepsilon_{1}}$ and $h_{\varepsilon_{2}}$ jointly occur with symbol $\varepsilon_{1}=\widehat{\varepsilon}_{1} \neq 1$, symbol $\varepsilon_{2}=\widehat{\varepsilon}_{2} \neq 1$, and $\widehat{\varepsilon}_{1} \neq \widehat{\varepsilon}_{2}$. Since the error probability in a Rayleigh fading channel is $1 /(2+\mathrm{SNR})$ [14], the probability of error caused by the two different interfering symbols is given by

$$
\begin{aligned}
A_{1} & =\frac{1}{2+\frac{S}{N_{0} T+x}}+\frac{1}{2+\frac{S}{N_{0} T+y}} \\
& \approx \frac{2 N_{0} T+x+y}{S}, \quad \frac{S}{N_{0} T} \gg 2
\end{aligned}
$$

where

$$
\begin{array}{ll}
S & \text { power of a useful signal; } \\
N_{0} T & \text { noise variance; } \\
x \text { and } y & \text { variances of two interfering symbols (or users), } \\
& \text { respectively, from adjacent cells. }
\end{array}
$$

Note that either $S / x$ or $S / y$ is always much greater than one.

Now suppose two events $h_{\varepsilon_{1}}$ and $h_{\varepsilon_{2}}$ jointly occur with $\varepsilon_{1}=$ $\widehat{\varepsilon}_{1} \neq 1, \varepsilon_{2}=\widehat{\varepsilon}_{2} \neq 1$, and $\widehat{\varepsilon}_{1}=\widehat{\varepsilon}_{2}$. Then, the probability of error caused by the two identical symbols and a noninterfering symbol is given by

$$
\begin{aligned}
A_{2} & =\frac{1}{2+\frac{S}{N_{0} T+x+y}}+\frac{1}{2+\frac{S}{N_{0} T}} \\
& \approx \frac{2 N_{0} T+x+y}{S}, \quad \frac{S}{N_{0} T} \gg 2 .
\end{aligned}
$$

It is clear from (23b) and (24b) that the two error probabilities are approximately the same. However, when one of the interfering symbols is from the intracell, $S$ and $x$ should be comparable. Both (23a) and (24a) are plotted in Fig. 4. It can be seen that when $S / x$ is small (i.e., the interfering symbol from intracell), $A_{2}$ is only $10 \%$ smaller than $A_{1}$. However, when $S / x$ is large (i.e., the interfering symbol possibly from an adjacent cell), both $A_{2}$ and $A_{1}$ are very close. Therefore, $A_{2}$ is a good approximation of $A_{1}$. Note that it is impossible for the two interfering symbols to be from intracell, since only a single hit is considered in any one cell. The above approximation can be applied to the joint occurrence of more than two single-hit events.

It is assumed that random variables $y_{1}, y_{2}$, and $y_{3}$ represent the number of hits from the cell of interest and the second and third layers of cells, respectively, for $N=3$, whereas for $N=7$, the random variables $y_{1}$ and $y_{4}$ are assumed to be the numbers of hits from the cell of interest and the third layer of cells, respectively. Note that $y_{1} \leq 1, y_{2} \leq 6, y_{3} \leq 6$, and $y_{4} \leq 6$. Also, it is assumed that random variables $x_{1}, x_{2}, x_{3}$, and $x_{4}$, corresponding to $y_{1}, y_{2}$, $y_{3}$, and $y_{4}$, respectively, represent the numbers of hits, which have the same symbols as the reference symbol $(\varepsilon=1)$ and $x_{1} \leq y_{1}$, $x_{2} \leq y_{2}, x_{3} \leq y_{3}$, and $x_{4} \leq y_{4}$.

Therefore, there are two cases for error probability.

1) For those branches $(M-1)$ that have no interfering symbol passing, the error probability is given by

$$
\begin{aligned}
P\left(\varepsilon \neq \varepsilon_{i}\right) & \begin{cases}\sum_{y_{1}=0}^{1} \sum_{y_{2}=0}^{6} \sum_{y_{3}=0}^{6} P_{e}\left(y_{1}, y_{2}, y_{3}\right) P_{h}\left(y_{1}, y_{2}, y_{3}\right), & N=3 \\
\sum_{y_{1}=0}^{1} \sum_{y_{4}=0}^{6} P_{e}\left(y_{1}, y_{4}\right) P_{h}\left(y_{1}, y_{4}\right), & N=7\end{cases}
\end{aligned}
$$

where $P_{e}\left(y_{1}, y_{2}, y_{3}\right)$ and $P_{h}\left(y_{1}, y_{2}, y_{3}\right)$ are the error probability and probability of the joint occurrence, respectively, of $\left(y_{1}, y_{2}, y_{3}\right)$ for $N=3$ [see Fig. 2(b)]. $P_{e}\left(y_{1}, y_{4}\right)$ and $P_{h}\left(y_{1}, y_{4}\right)$ are the error probability and probability of the joint occurrence of $\left(y_{1}, y_{4}\right)$, respectively, for $N=7$ [see Fig. 2(a)]. When $N=3$, those probabilities are given by

$$
\begin{aligned}
& P_{h}\left(y_{1}, y_{2}, y_{3}\right) \\
& \quad=\left(\begin{array}{c}
6 \\
y_{2}
\end{array}\right)\left(\begin{array}{c}
6 \\
y_{3}
\end{array}\right) P_{h}^{y_{1}+y_{2}+y_{3}}\left(1-P_{h}\right)^{13-y_{1}-y_{2}-y_{3}}
\end{aligned}
$$






Fig. 4. Equations (23a) and (24a) as a function of $S / x$.

where $P_{h}$ is given by (16) and

$$
\begin{aligned}
& P_{e}\left(y_{1}, y_{2}, y_{3}\right) \\
& \quad=\sum_{x_{1}=0}^{y_{1}} \sum_{x_{2}=0}^{y_{2}} \sum_{x_{3}=0}^{y_{3}} \hat{P}_{e}\left(x_{1}, x_{2}, x_{3}\right) \hat{P}_{h}\left(x_{1}, x_{2}, x_{3}\right)
\end{aligned}
$$

where

$$
\begin{aligned}
\hat{P}_{h}\left(x_{1}, x_{2}, x_{3}\right)= & \left(\begin{array}{l}
y_{2} \\
x_{2}
\end{array}\right)\left(\begin{array}{l}
y_{3} \\
x_{3}
\end{array}\right)\left(\frac{1}{M}\right)^{x_{1}+x_{2}+x_{3}} \\
& \cdot\left(1-\frac{1}{M}\right)^{y_{1}+y_{2}+y_{3}-x_{1}-x_{2}-x_{3}} \\
\hat{P}_{e}\left(x_{1}, x_{2}, x_{3}\right)= & \frac{1}{2+r_{1}\left(x_{1}, x_{2}, x_{3}\right)}
\end{aligned}
$$

where

$$
\begin{gathered}
r_{1}\left(x_{1}, x_{2}, x_{3}\right) \\
=\frac{S_{1}+S_{2}+x_{1} \sigma_{1}^{2}+x_{2} \sigma_{2}^{2}(\gamma, 1)+x_{3} \sigma_{2}^{2}(\gamma, 2)}{N_{0} T_{s}} \\
=\frac{\bar{E}_{b}}{N_{0}}\left(\log _{2} M\right)\left\{\left[1+\alpha x_{1}+\alpha x_{2} \xi(\gamma, 1)\right.\right. \\
\left.\left.+\alpha x_{3} \xi(\gamma, 2)\right] L+1\right\} / 2
\end{gathered}
$$

where $S_{1}, S_{2}, \sigma_{1}^{2}$, and $\sigma_{2}^{2}(\gamma, \cdot)$ are given by (11), (13), (15), and (19), respectively. $\bar{E}_{b}=2 P_{i} T_{b} \rho_{0}=$ $2 P_{i} T_{s} \rho_{0} \log _{2} M$ is the average energy per coded bit. $\alpha=P_{1} / P_{0}$ is the ratio of the received nonreference user power to the received reference user power. $\xi(\gamma, x)$ is given by Table I.

When $N=7$, in (25), we have

$$
\begin{aligned}
& P_{h}\left(y_{1}, y_{4}\right)=\left(\begin{array}{c}
6 \\
y_{4}
\end{array}\right) P_{h}^{y_{1}+y_{4}}\left(1-P_{h}\right)^{7-y_{1}-y_{4}} \\
& P_{e}\left(y_{1}, y_{4}\right)=\sum_{x_{1}=0}^{y_{1}} \sum_{x_{4}=0}^{y_{4}} \hat{P}_{e}\left(x_{1}, x_{4}\right) \hat{P}_{h}\left(x_{1}, x_{4}\right)
\end{aligned}
$$

where

$$
\begin{aligned}
& \hat{P}_{h}\left(x_{1}, x_{4}\right)=\left(\begin{array}{l}
y_{4} \\
x_{4}
\end{array}\right)\left(\frac{1}{M}\right)^{x_{1}+x_{4}}\left(1-\frac{1}{M}\right)^{y_{1}+y_{4}-x_{1}-x_{4}} \\
& \hat{P}_{e}\left(x_{1}, x_{4}\right)=\frac{1}{2+r_{2}\left(x_{1}, x_{4}\right)}
\end{aligned}
$$

where

$$
\begin{aligned}
r_{2}\left(x_{1}, x_{4}\right) & =\frac{S_{1}+S_{2}+x_{1} \sigma_{1}^{2}+x_{4} \sigma_{2}^{2}(\gamma, 3)}{N_{0} T_{s}} \\
& =\frac{\bar{E}_{b}}{N_{0}}\left(\log _{2} M\right)\left\{\left[1+\alpha x_{1}+\alpha x_{4} \xi(\gamma, 3)\right] L+1\right\} / 2 .
\end{aligned}
$$

2) For the branch (only one branch) that has interfering symbols passing, the discussion of the error probability is also the same as the above, only by substituting $r_{1}\left(x_{1}, x_{2}, x_{3}\right)$ with $r_{3}\left(x_{1}, x_{2}, x_{3}\right)$ and $r_{2}\left(x_{1}, x_{4}\right)$ with $r_{4}\left(x_{1}, x_{4}\right)$, given by (36) and (37), shown at the bottom of the next page.

Once the SER $P_{s}$ after hard decision has been obtained, the corresponding BER $P_{b}$ is given by

$$
P_{b}=\frac{M}{2(M-1)} P_{s} .
$$

The above expression assumes that given a symbol error, all $M-1$ erroneous symbols are equally likely to be chosen. This assumption is valid because of the orthogonality of the MFSK signals and the statistical properties of the disturbance (noise + multiaccess interference + adjacent cell interference).

\section{B. Error Probability After Decoding}

Reed-Solomon codes are nonbinary, linear, cyclic symbolerror-correcting block codes. The length of an RS code is $n=$ $M-1 M$-ary symbols, $m$ of which are information symbols and the remaining $n-m$ of which are redundancy. This code is referred to as an $\operatorname{RS}(n, m)$ code. It can correct up to $[(n-m) / 2]$ 


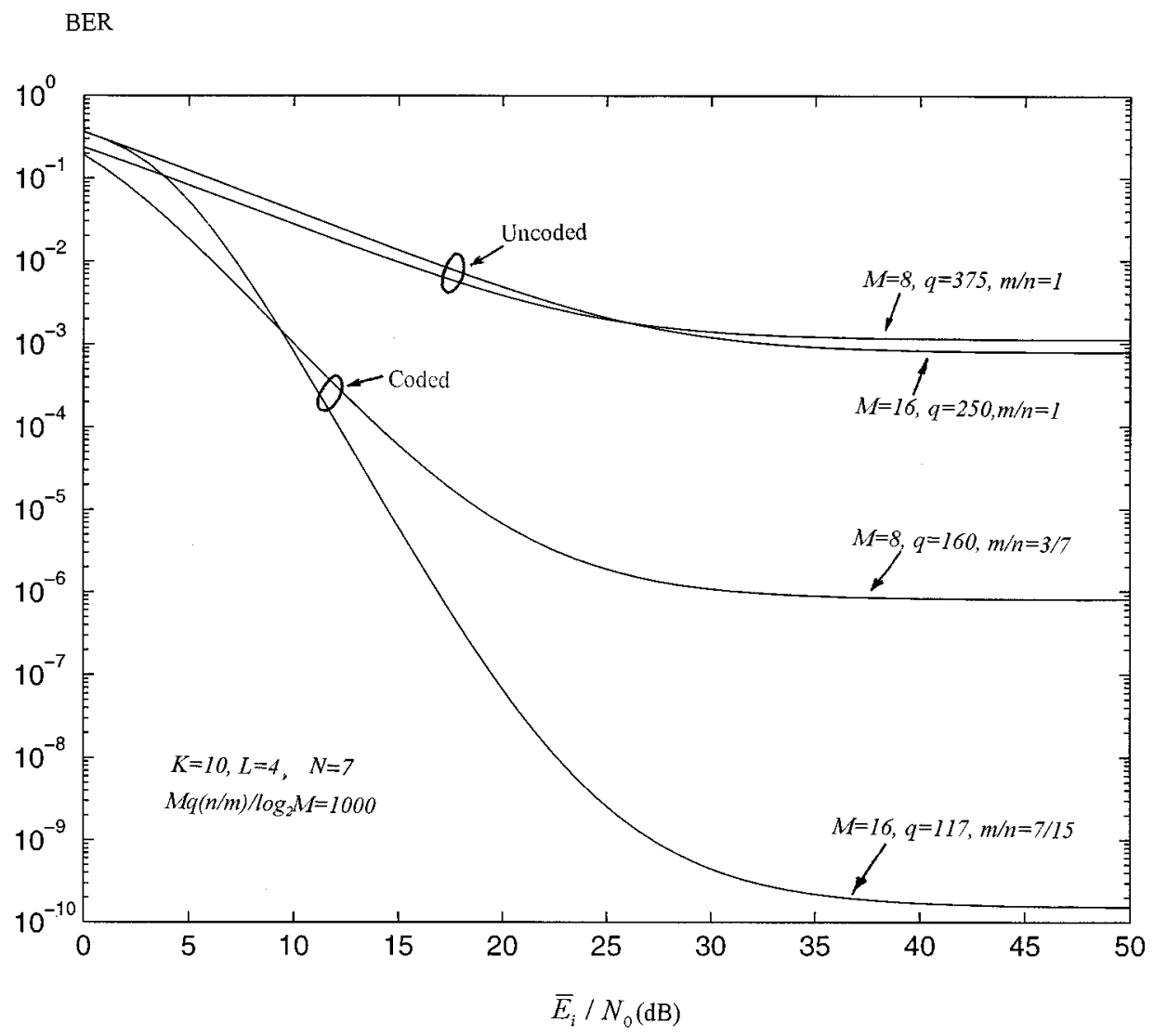

Fig. 5. BER with and without RS coding for fixed system bandwidth.

symbol errors, where $[x]$ denotes the largest integer contained in $x$. We have selected $m=(M-2) / 2$ so that the coding rate $m / n$ is nearly $1 / 2$ and the code can correct up to $t=M / 4$ symbol errors. When decoding of RS codes with hard decision is employed, the symbol error probability $P_{\mathrm{SC}}$ after RS decoding is well approximated by [14], given

$$
P_{\mathrm{SC}}=\frac{1}{n} \sum_{i=t+1}^{n} i\left[\begin{array}{l}
n \\
i
\end{array}\right] P_{s}^{i}\left[1-P_{s}\right]^{n-i}
$$

where

$t \quad M / 4$

n $\quad M-1$;

$P_{s} \quad$ symbol error probability before decoding.

The above expression assumes that the symbol errors before decoding occur independently. This assumption is valid when an interleaving/deinterleaving technique is used even though a hit or more hits may be possible. The $P_{\mathrm{bc}}$ after decoder is approximated by substituting in $P_{b}$ and $P_{s}$ by $P_{\mathrm{bc}}$ and $P_{\mathrm{sc}}$.

$$
\begin{aligned}
r_{3}\left(x_{1}, x_{2}, x_{3}\right) & =\frac{S_{1}+S_{2}+x_{1} \sigma_{1}^{2}+x_{2} \sigma_{2}^{2}(\gamma, 1)+x_{3} \sigma_{2}^{2}(\gamma, 2)}{N_{0} T_{s}+\left(y_{1}-x_{1}\right) \sigma_{1}^{2}+\left(y_{2}-x_{2}\right) \sigma_{2}^{2}(\gamma, 1)+\left(y_{3}-x_{3}\right) \sigma_{2}^{2}(\gamma, 2)} \\
& =\frac{1+\alpha\left(1-\frac{1}{L+1}\right)\left[x_{1}+x_{2} \xi(\gamma, 1)+x_{3} \xi(\gamma, 2)\right]}{\frac{2}{\bar{E}_{b}}\left(\log _{2} M\right)(L+1)}+\alpha\left(1-\frac{1}{L+1}\right)\left[\left(y_{1}-x_{1}\right)+\left(y_{2}-x_{2}\right) \xi(\gamma, 1)+\left(y_{3}-x_{3}\right) \xi(\gamma, 2)\right] \\
r_{4}\left(x_{1}, x_{4}\right)= & \frac{S_{1}+S_{2}+x_{1} \sigma_{1}^{2}+x_{4} \sigma_{2}^{2}(\gamma, 3)}{N_{0} T_{s}+\left(y_{1}-x_{1}\right) \sigma_{1}^{2}+\left(y_{4}-x_{4}\right) \sigma_{2}^{2}(\gamma, 3)} \\
= & \frac{1+\alpha\left(1-\frac{1}{L+1}\right)\left[x_{1}+x_{4} \xi(\gamma, 3)\right]}{\frac{2}{\bar{E}_{b}}\left(\log _{2} M\right)(L+1)}+\alpha\left(1-\frac{1}{L+1}\right)\left[\left(y_{1}-x_{1}\right)+\left(y_{4}-x_{4}\right) \xi(\gamma, 3)\right]
\end{aligned}
$$




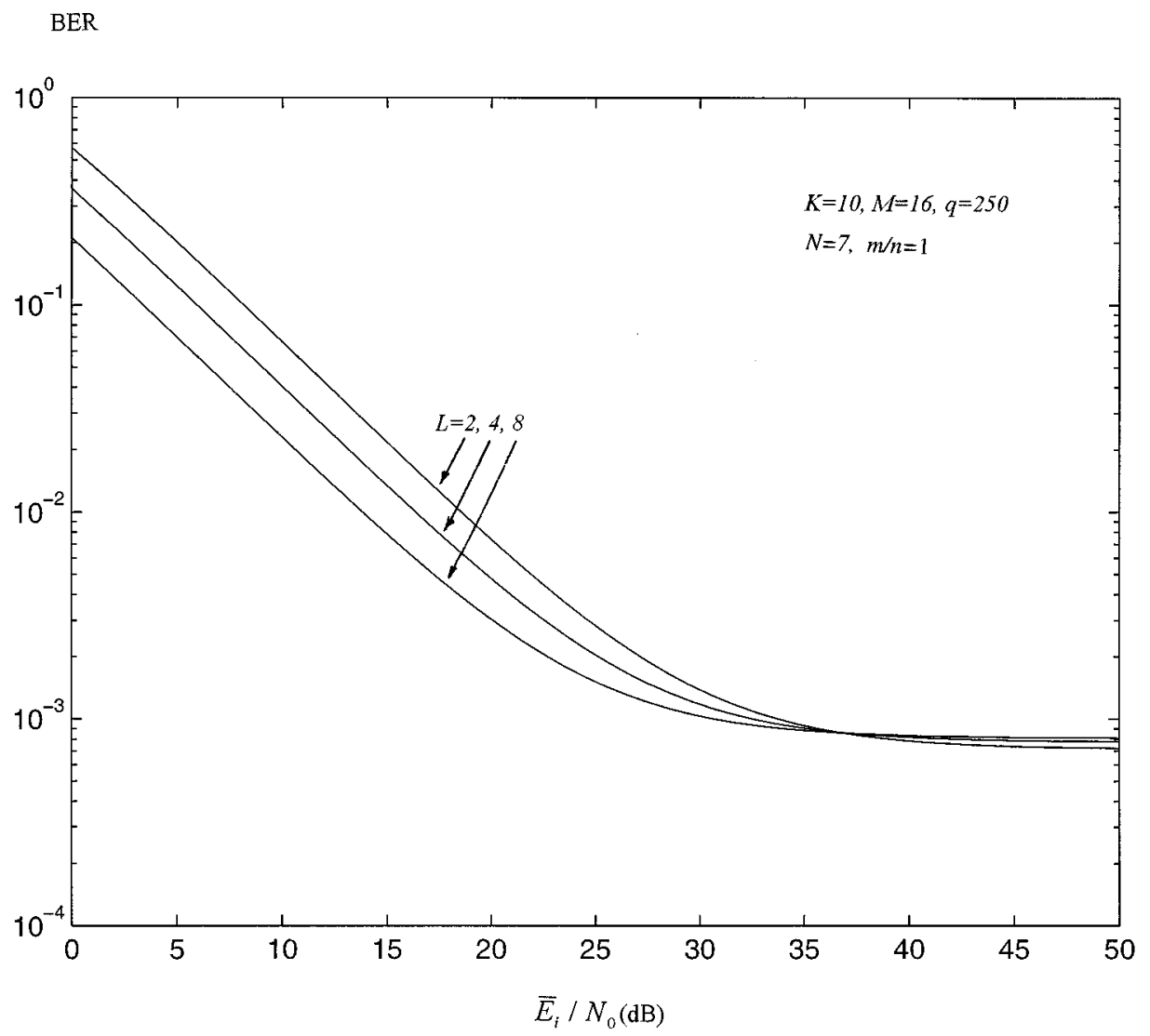

Fig. 6. Effect of number of paths $(L)$ on uncoded BER.

\section{NUMERICAL RESULTS}

The BERs of one-hop/symbol cellular FHMA systems with MFSK modulation, noncoherent square-law envelope detection, and RS coding for a multipath Rayleigh channel are now evaluated. Representative numerical results are presented. It is assumed that the value of the propagation exponent equals three (or $\gamma=3$ ) so that $\xi(\gamma, x)=0.013,0.0033$ and 0.0023 for $x=1,2,3$, respectively. Unless indicated otherwise, we assume $\alpha=1$. For a fair comparison of the BERs of uncoded and coded systems, it is assumed that these systems have the same information bit rate $R_{i}$, the same system bandwidth $B$, given by (2), and the same energy per uncoded bit $\bar{E}_{i}\left(\bar{E}_{i}=\bar{E}_{b} n / m\right)$.

First, in Fig. 5, the RS-coded BERs are illustrated for different $M$, given a fixed system bandwidth $B$, given by (2). Also, uncoded $(n / m=1)$ BERs are shown for comparison. As denoted in (16) and (26), the asymptotic uncoded BER increases as $q$ decreases (or as $M$ increases for fixed values of $\left(M q / \log _{2} M\right)$, whereas the performance advantage obtained by using RS coding becomes larger when $M$ increases. This is because the RS code error-correcting capability increases when $M$ is increased.

The effect of the number of paths $L$ on the uncoded BER performance is considered in Fig. 6. It is seen that for $\bar{E}_{i} / N_{0}<30 \mathrm{~dB}$, additive Gaussian noise is the dominating disturbance. The BER performance improves with increasing $L$, the useful variance due to the $L$ paths of the reference user increases with $L$, whereas the noise variance does not depend on $L$. For $\bar{E}_{i} / N_{0} \rightarrow \infty$, the multiple-access and adjacent-cell interference is the dominating disturbance. In this case, the BER is only weakly dependent on the number of paths $(L)$, because both the useful variance and the interference variance are essentially proportional with $L$. As described in Section IV for asymptotic performance, it is clear from (36) and (37) that the BER is almost independent of $L$ for $L>1$. This is in contrast with DS systems, the performance of which is degraded as $L$ increases. Note that this discussion is not involved with any diversity (or Rake receiver).

The asymptotic BER for FHMA systems with RS coding is shown in Fig. 7 for the reuse factor of three $(N=3)$ as a function of the nonreference user to reference user power ratio $\alpha$ for a given system bandwidth. For comparison, the BER of a DS code-division multiple-access (CDMA) system with the same system bandwidth (processing gain $\hat{N}=1000$ ) is also shown in Fig. 7. Note that the reuse factor of the DS-CDMA system is unitary. A powerful coding Golay $(23,12)$ code with half-code rate and equal gain combining of the second order for the DS-CDMA system [1] are used. Its uncoded error probability is given by [1, (19)] with signal-to-noise ratio $r_{c}=3 \hat{N} /[2 K L(1+\xi(\gamma)]$, where $\xi(\gamma)$ stands for the effect of adjacent- cell interference and $\xi(\gamma)=0.9$ [1] when the propagation exponent is three. Then, the coded BER is given by [1, (18)], with $n=23$ and $t=3$ for Golay (23, 12) code, which can correct three errors in one block code. It can be seen that when $\alpha$ is very small, the DS-CDMA system outperforms the FHMA system. However, when $\alpha$ is very 




Fig. 7. Asymptotic BER with RS coding for fixed system bandwidth.

large, the performance of DS-CDMA degrades dramatically, whereas the FHMA system can keep acceptable performance. Therefore, the frequency-hopping system is more robust than the DS-CDMA system in overcoming the near/far problem.

Finally, Fig. 8 illustrates the BER of a cellular FHMA system as a function of the number of active users per cell for different values of frequency reuse factor $N$ when the system bandwidth is fixed $(q N=1000)$. It is seen from this figure that for a given BER, a smaller value of $N$ provides larger overall capacity of the cellular system. Although a smaller value of $N$ causes more adjacent-cell interference, it increases the total number $q$ of hopping frequencies per cell, which decreases the hit probability (16) more and, therefore, reduces BER tremendously. Basically, this conclusion is consistent with the general conclusion of cellular FDMA systems, where system capacity increases with a decreasing of the number of cells in a cellular cluster.

\section{CONCLUSION}

In this study, the BER performance of one hop per symbol FHMA with MFSK modulation, noncoherent envelope detection, and RS coding has been investigated for cellular applications. The following results have been obtained.
1) By employing RS coding, a significant improvement in BER performance is obtained when the number of MFSK signals increases.

2) The FHMA system is superior to a DS-CDMA system in overcoming the near/far problem.

3) In order to have high capacity of the cellular FHMA system, a small value of the frequency reuse factor should be used.

\section{APPENDIX \\ MulTiPATH TERMS}

Let us consider the output of the MFSK demodulator branch, which is the closest in frequency to the branch with $\varepsilon=b_{i}^{(\lambda)}$. Suppose the frequency offset of the closest branch is $2 \Delta$ and the delay and phase of the $l$ th path of the reference user are $\tau$ and $\phi$, respectively. The output of the closest branch is $|X|^{2}$, where $X$ is given by

$$
\begin{aligned}
X= & \int_{\tau}^{T_{s}} \sqrt{2 P_{i}} \beta_{i l} \cos \left[\left(2 \pi f_{0}+2 \pi b_{i}^{(\lambda)} \Delta\right) t+\Phi\right] \\
& \cdot 2 \exp \left[j\left(2 \pi f_{0}+2 \pi b_{i}^{(\lambda)} \Delta+2 \pi \cdot 2 \Delta\right) t\right] d t .
\end{aligned}
$$

Removing the high-frequency components, $X$ reduces to

$$
X=\sqrt{2 P_{i}} \beta_{i l} \int_{\tau}^{T_{s}} \exp [j 2 \pi \cdot 2 \Delta t-j \Phi] d t .
$$

The real part of (A2) is given by

$$
\begin{aligned}
\operatorname{Re}\{X\}= & \sqrt{2 P_{i}} \beta_{i l} \int_{\tau}^{T_{s}}[\cos (2 \pi \cdot 2 \Delta t) \cos \Phi \\
= & \sqrt{2 P_{i}} \beta_{i l} \frac{1}{2 \pi \cdot 2 \Delta} \\
& \cdot\left\{\left[\sin (2 \pi \cdot 2 \Delta t) \sin \left(2 \pi \cdot 2 \Delta T_{s}\right)-\sin (2 \pi \cdot 2 \Delta \tau)\right] \cos \Phi\right. \\
& \left.-\left[\cos \left(2 \pi \cdot 2 \Delta T_{s}\right)-\cos (2 \pi \cdot 2 \Delta \tau)\right] \sin \Phi\right\}
\end{aligned}
$$

where $\sin \left(2 \pi \cdot 2 \Delta T_{s}\right)=0$ and $\cos \left(2 \pi \cdot 2 \Delta T_{s}\right)=1$ because $2 \Delta T_{s}=n_{o}$ is an integer. Therefore, $\operatorname{Re}\{X\}$ reduces to

$$
\begin{aligned}
\operatorname{Re}\{X\}= & -\sqrt{2 P_{i}} \beta_{i l} \frac{1}{2 \pi \cdot 2 \Delta} \\
& \cdot\{\sin (2 \pi \cdot 2 \Delta \tau) \cos \Phi+[1-\cos (2 \pi \cdot 2 \Delta \tau)] \sin \Phi\} .
\end{aligned}
$$

It is assumed that the delay $\tau$ and phase $\Phi$ are uniformly distributed in $\left[0, T_{s}\right]$ and $[0,2 \pi]$, respectively. The variance of $\operatorname{Re}\{X\}$ is given by

$$
\begin{aligned}
\sigma_{X}^{2}=2 P_{i} E\left[\beta_{i}^{2}\right] \frac{1}{(2 \pi \cdot 2 \Delta)^{2}} E\{[\sin (2 \pi \cdot 2 \Delta \tau) \cos \Phi \\
\left.\quad+[1-\cos (2 \pi \cdot 2 \Delta \tau)] \sin \Phi]^{2}\right\} \\
=2 P_{i} 2 \rho \frac{1}{(2 \pi \cdot 2 \Delta)^{2}}\left\{E\left[\sin ^{2}(2 \pi \cdot 2 \Delta \tau)\right] \cdot E\left[\cos ^{2} \Phi\right]\right. \\
\left.\quad+E\left[1+\cos ^{2}(2 \pi \cdot 2 \Delta \tau)\right] E\left[\sin ^{2} \Phi\right]\right\} \\
=2 P_{i} 2 \rho \frac{1}{(2 \pi \cdot 2 \Delta)^{2}}\left\{\frac{1}{2} \cdot \frac{1}{2}+\left(1+\frac{1}{2}\right) \cdot \frac{1}{2}\right\} \\
=\frac{P_{i} \rho}{(\pi \cdot 2 \Delta)^{2}}=\frac{P_{i} \rho \cdot T_{s}^{2}}{\left(\pi \cdot 2 \Delta T_{s}\right)^{2}}=\frac{P_{i} \rho \cdot T_{s}^{2}}{\pi^{2} \cdot n_{0}^{2}}
\end{aligned}
$$


BER

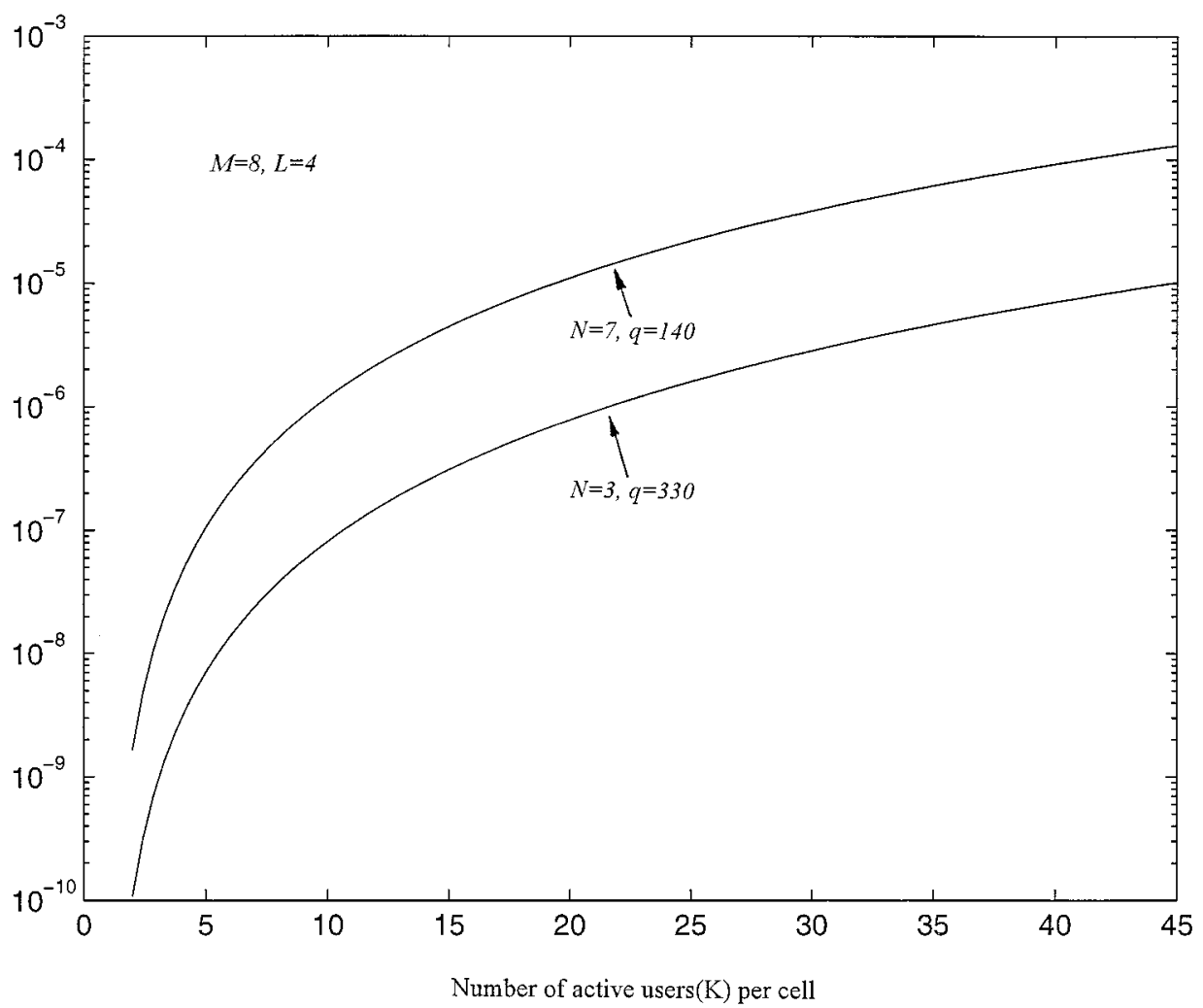

Fig. 8. BER comparison of $N=3$ and $N=7$ for fixed system bandwidth.

where $n_{0}=2 \Delta T_{s}$ is an integer. Similarly, the variance of the imaginary part of (A2) is also given by (A5).

\section{REFERENCES}

[1] J. Wang and L. B. Milstein, "CDMA overlay situations for microcellular mobile communications," IEEE Trans. Commun., vol. 43, pp. 603-614, Feb. 1995.

[2] E. A. Geraniotis and M. B. Pursley, "Error probability for slow-frequency-hopping spread-spectrum multiple-access communications over fading channels," IEEE Trans. Commun., vol. COM-30, pp. 996-1009, May 1982.

[3] W. E. Stark, "Coding for frequency hopped spread spectrum communications with partial-band interference-Part II: Coded performance," IEEE Trans. Commun., vol. COM-33, pp. 1045-1057, Oct. 1985.

[4] T. C. Wu, C. C. Chao, and K. C. Chen, "Capacity of synchronous coded DS SFH and FFH spread spectrum multiple access for wireless local communications," IEEE Trans. Commun., vol. 45, pp. 200-212, Feb. 1997.

[5] D. V. Sarwate and M. B. Pursley, "Hopping patterns for frequency hopped multiple access communications," in Proc. Int. Conf. Communications, 1987, pp. 7.4.1-7.4.5.

[6] A. Lam and D. V. Sarwate, "Time-hopping and frequency-hopping multiple-access packet communications," IEEE Trans. Commun., vol. 38, pp. 875-888, June 1990.

[7] - "Multiple-user interference in FHMA-DPSK spread-spectrum communications," IEEE Trans. Commun., vol. COM-33, pp. 1-12, Jan. 1986.

[8] M. Chiani, "Error probability for block codes over channels with block interference," IEEE Trans. Inform. Theory, vol. 44, pp. 2998-3008, Nov. 1998.

[9] M. Chiani, A. Conti, E. Agrati, and O. Andrisano, "Outage evaluation for slow frequency hopping mobile radio systems," IEEE Trans. Commun., vol. 47, pp. 1865-1874, Dec. 1999.

[10] D. Lim and L. Hanzo, "The probability of multiple correct packet reception in coded synchronous frequency hopped spread spectrum networks," IEEE Trans. Commun., vol. 47, pp. 1227-1232, Aug. 1999.
[11] C. W. Baum and M. B. Pursley, "A decision-theoretic approach to the generation of side information in frequency hop multiple access communications," IEEE Trans. Commun., vol. 43, pp. 1768-1777, Mar. 1995.

[12] J. Wang and M. Moeneclaey, "Multihops/symbol FFH-SSMA with MFSK modulation and Reed-Solomon coding for indoor radio," IEEE Trans. Commun., vol. 41, pp. 793-801, May 1993.

[13] — , "Hybrid DS/SFH-SSMA with predetection diversity and coding over indoor radio multipath Rician fading channels," IEEE Trans. Commun., vol. 40, pp. 1654-1662, Oct. 1992.

[14] J. G. Proakis, Digital Communications. New York: McGraw-Hill, 1995.

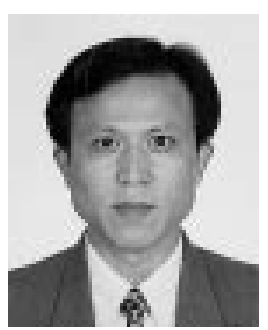

Jiangzhou Wang (M'91-SM'94) received the B.S. and M.S. degrees in electrical engineering from Xidian University, Xian, China, in 1983 and 1985, respectively, and the Ph.D. degree (with greatest distinction) from the University of Ghent, Belgium, in 1990, also in electrical engineering.

From 1990 to 1992, he was a Postdoctoral Fellow at the University of California, San Diego, where he worked on research and development of cellular CDMA systems. From 1992 to 1995, he was a Senior System Engineer with Rockwell International Corporation, Newport Beach, CA, where he worked on the development and system design of wireless communications. Since 1995, he has been an Associate Professor at the University of Hong Kong, teaching and conducting research in wireless mobile and spread-spectrum communications. He has one U.S. patent for the GSM system.

Dr. Wang is an Editor for the IEEE TRANSACTIONS ON COMMUNICATIONS and a Guest Editor for the IEEE JOURNAL ON SELECTED AREAS IN COMMUNICATIONS. 